\title{
Challenges and Countermeasures of Managing Private-owned Traditional Chinese Medical Hospital in Tianjin: A Case Study
}

\author{
Yizhi Zhang \\ Tianjin University of Traditional Chinese Medicine \\ TJUTCM \\ Tian jin, China \\ 459640201@qq.com
}

\author{
Qiang $\mathrm{He}^{*}$ \\ Tianjin University of Traditional Chinese Medicine \\ TJUTCM \\ Tian jin, China \\ heqiangmail@126.com
}

\author{
Xiaofang Wang \\ Tianjin University of Traditional Chinese Medicine \\ TJUTCM \\ Tianjin, China \\ 1468630344@qq.com
}

\begin{abstract}
Based on the actual situation of traditional Chinese medical hospital as well as the research of domestic and foreign literature, this paper discusses Tianjin's traditional Chinese medical hospitals. With the help of SWOT analysis, on-the-spot investigation and questionnaire surveys, we try to understand the problems of the traditional Chinese medicine. Besides, this paper provides references to the market development of traditional Chinese medical hospital, with the consideration of the patients' outpatient services, doctor resources, and medical driving factors and so on.
\end{abstract}

Keywords-Tianjin Chinese traditional medicine; privateowned hospital; management challenges; SWOT analysis

Since the reform and opening-up, Chinese economy has developed rapidly and the health-care market has been expanding dramatically.[1] However, compared with public and foreign hospitals, the development of private-owned hospitals is accompanied with lots of challenges. The related researches of private hospitals are rather few and the corresponding theories aren't systematic, not mentioning the research of Tianjin's traditional Chinese medical hospitals. In fact, it is on the turning-point of its thirty years development history, with both opportunities and challenges.[2] Compared with public hospitals, private-operated hospital is a new medical economic entity, which is invested by individuals or private associations. Few are non-profit organizations with the government subsidies.[3] The main characteristics of privateowned hospitals are independent decision-making, management and self-financing.

This case study would like to analyses the current operation situation of Tianjin's private traditional Chinese medical hospitals and find out the existing problems. [4]We will discuss it from several aspects including organizational structure, financial management, doctor resources, patient sources and competitors. In order to help them overcome difficulties, this paper will try to find a healthy and sustainable development path for Tianjin's private traditional Chinese medical hospitals and offer suggestions. Also, it would be the reference for other similar private-owned hospitals.

\section{Development Situation of Tianjin's Traditional CHINESE Medical Hospitals}

\section{A. Problems of the Hospitals}

Along with the market economy, the amount of private hospitals has seen a geometric growth. There were only 13 private-owned hospitals in Tianjin in 2001, while there are more than 5600 now. The profits of traditional Chinese medical hospitals have been declined these years with the severe competition. In 2015 and 2016, profits fell about 13\% and 26\% respectively. Hence, the development is not optimistic.

TABLE I. ST ATIST ICAL T ABLE OF LIFTING RATIO OF TRADITIONAL CHINESE MEDICAL HOSPIT AL SIN RECENT YEARS

\begin{tabular}{|l|c|c|}
\hline & outpatient volume & turnover \\
\hline 2013 & & \\
\hline 2014 & $\uparrow 1.66 \%$ & \\
\hline 2015 & $\downarrow 7.41 \%$ & $\uparrow 2.4 \%$ \\
\hline 2016 & $\downarrow 18.91 \%$ & $\uparrow 3.8 \%$ \\
\hline
\end{tabular}

The decrease of the outpatient volume leads to the decrease of the overall profit of the hospitals, although the turnover increases slightly because of the increase of drugs' prices. However, considering that the profits of drugs also decrease year by year, the profits is decreasing gradually. Secondly, in order to save time and costs, most of hospitals make tisane by machine now. Although it may accord with the requirements of 
the quality control of the Health Bureau, the effects of the tisane are not good enough. Hence, traditional Chinese medical hospital usually chooses to make the tisane manually, and it leads to the higher cost of expenditure as it may consume more time and labor. What's more, considering that some of them are non-profitable hospitals, nearly all the traditional Chinese medical hospitals have to face with the problems including increasing rent, coal or water leaking and increasing operating costs.

While the twenty-first century is an era of internet, it gives us the power to share information and unite millions of people from the whole world. The traditional Chinese medical hospital rely on praises from mouth to mouth without any media publicity.[5] Hence, the new pattern cannot go in if the old one doesn't change. The diagnosis of traditional Chinese medicine is too simple to face the fierce competition. And the problem is that, how to develop and expand the market under such a situation.

\section{B. Issues about Doctors}

Tianjin's old TCM doctors who are in clinic are too old and dying off. Because of age, many old doctors tend to decrease the number of appointments which leads to the decline of outpatient visits and the shortage of doctor resources. Doctors are too old to move so that the spending spurs because old doctors need picking up service. To save patients' time, doctors are equipped with assistants which results in increased outlays. Hospitals don't possess advantages comparing with thirty years ago due to doctor's multi-sited license and the rehire of retired doctors. Besides, by reason of strained relationship between doctors and patients, hospitals lack young doctor resources to build a talent team.

\section{Issues about Patients}

TABLE II. ST ATIST ICS ABOUT DRIVING FACT ORS OF PATIENT S SEEKING MEDiCAL AdVICE

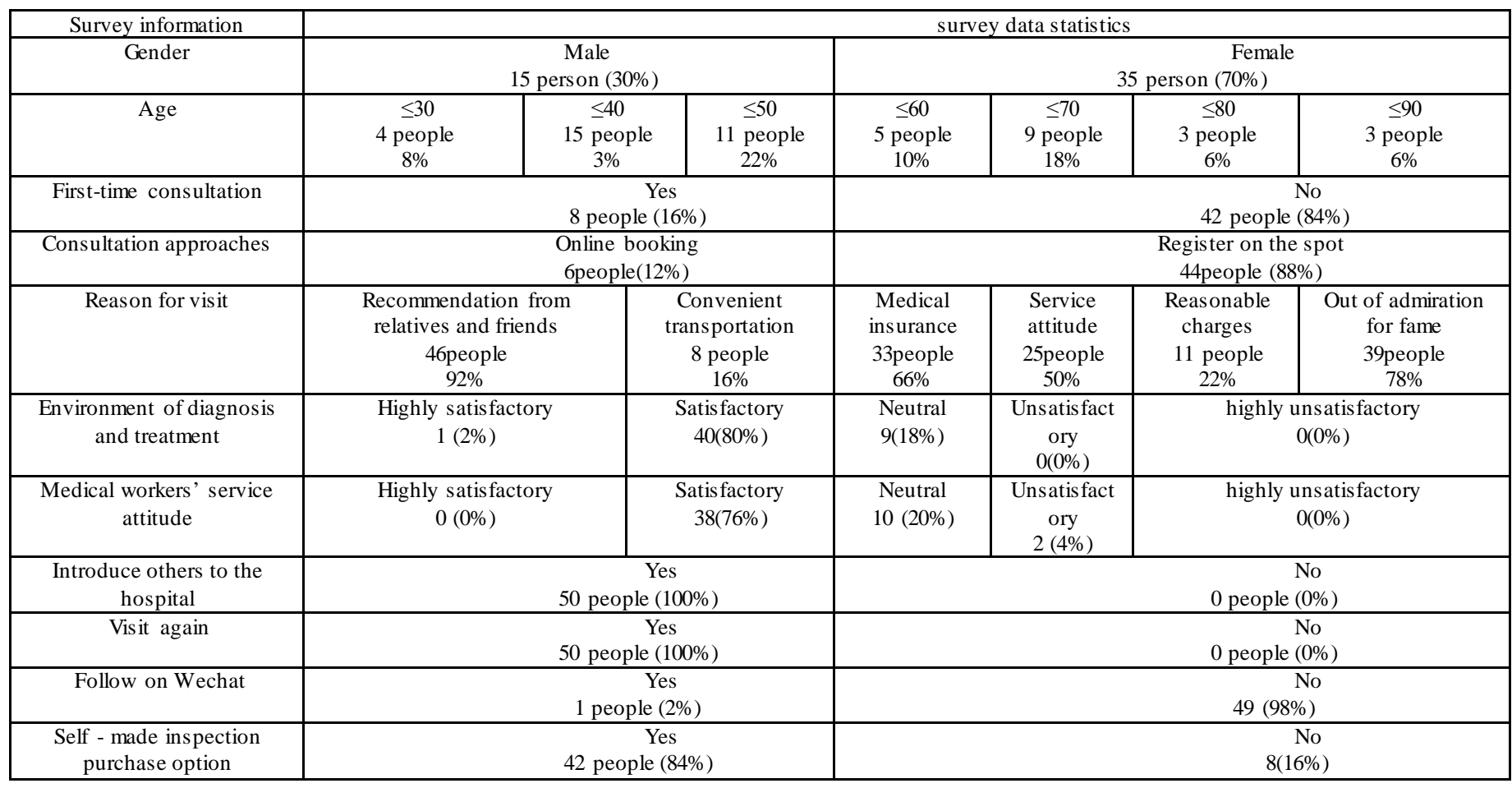

According to the survey, most patients in the senior Chinese medicine hospitals are not hospitalized for the first time, resulting a high satisfaction degree with the hospital with relatives and friends recommended. Medical insurance are the main reasons for the treatment of patients to the Tianjin hospital of Traditional Chinese Medicine. Most patients choose to register on the spot because online booking registration method has not yet fully popularized and they also expressed their willingness to come to Tianjin senior Chinese hospitals again for treatment and would like to recommend it to their relatives and friends. This shows that the overall service quality of Tianjin senior Chinese hospitals is ideal. However, the management system, publicity and promotion and the popularity of WeChat public platform can also be further improved to achieve the overall progress of Tianjin senior Chinese hospitals.

\section{SWOT ANalysis about Tianjin Old Chinese Hospitals}

\section{A. Advantages}

The government's strong support and preferable policies for medical care. The old Chinese medicine hospital is located in downtown which is a good location,. It has a long history with public praises. Special Chinese medicine decoction service, preferential price and good technology of massage, scrapping, cupping and acupuncture are all attracting. There are well-known domestic and foreign famous old TCM doctors, 
prestigious Chinese family descendants, and traditional Chinese and Western medicine experts.

\section{B. Disadvantages}

Firstly, there is still much room for service improvement. Secondly, poor management of employed personnel and processes are quite chaotic; thirdly, insufficient personnel training; fourthly, low popularity due to poor use of the internet. Less competitiveness because of no wards and single development projects. The average number of outpatient visit is around 200 numbers a day; the number of out-patient medical services will have a certain impact on the quality.

\section{Opportunities}

As of 2015, the number of people over the age of 60 in our country has reached 220 million, accounting for $16 \%$ of the total population. The aging population is suffering from the rapid growth in medical demand. public hospitals alone are insufficient to solve the problem, which offer the opportunity for .private hospitals There are many residential buildings near
Tianjin Old Chinese Medicine Hospital, which is the only one hospital in the community, the number of people getting medical treatment to take medical treatment to take medicine, the majority of patients over the age more than 40years, and sit-in doctor reputation. The exquisite differentiation of old experts, pharmacies pure quality Pieces, so that many patients with intractable diseases have been timely and effective treatment for fixed-point hospital in Tianjin Medicare.

\section{Threats}

In recent years, frequent disputes between doctors and patients and imperfect medical institutions have also had a great impact on the development of Tianjin Old Chinese Medicine Hospital. [6] The development of the hospital is very important. Failure to do a good job in the quality of the hospital may lead to doctor-patient disputes. The strong economic strength such as foreign medical care and competitors as well as advanced and technologically complete equipment has aggravated the threat. Furthermore, the introduction of talent in private hospitals is not as attractive as public hospitals.

TABLE III. SWOT ANALYTICAL DiAGRAM OF TIANJIN PRIVATE TRADITIONAL CHINESE MEDiCine HoSPIT AL

\begin{tabular}{|c|c|c|}
\hline $\begin{array}{l}\text { S (advantages): } \\
\text { 1.The overall image of the hospital is good } \\
\text { 2.Good geographical location } \\
\text { 3.Medical technical ambition } \\
\text { 4.Good national policy } \\
\text { 5.Good quality of drugs }\end{array}$ & \multirow[t]{2}{*}{ SWOT analysis } & $\begin{array}{l}\text { W(disadvantage): } \\
\text { 1.Service quality to be improved } \\
\text { 2.Medical forms are not diversified } \\
\text { 3.Single ward development project } \\
\text { 4.Lack of scientific management } \\
\text { 5.Lack of media publicity }\end{array}$ \\
\hline $\begin{array}{l}\text { O (opportunities): } \\
\text { 1.Adjacent residential area } \\
\text { 2.Promotion of medical environment } \\
\text { 3.Government recognition and support } \\
\text { 4.The aging of the population is severe } \\
\text { 5.Turnkey Support Via Vendor }\end{array}$ & & $\begin{array}{l}\text { T( threat): } \\
\text { 1.Foreign medical treatment } \\
\text { 2.Lack of doctors' resources } \\
\text { 3.Weak echelon construction } \\
\text { 4.Medical disputes } \\
\text { 5.Competitor }\end{array}$ \\
\hline
\end{tabular}

\section{Prepare Management Countermeasures of Tianjin PRIVATE TRADITIONAL CHINESE MEDICINE HOSPITAL}

\section{A. To Hold the Year of Hospital Management, Shaping Corporate Culture}

To Strengthen hospital management and improve fullservice quality, clearer job responsibilities should be established and staff be motivated.[7] Hospital staff incentive system should be introduced, so as to keep strictly the rules for reward and punishment. It can motivate staff to work with passion. Guided by the concept of scientific development, the old Chinese medicine hospital is more capable of creating an enterprise culture from four aspects: material culture, behavior culture, system culture and spiritual culture.

It should be implemented in the aspects of improving the hospital facilities environment, health care workers' words and deeds, their service attitude. To regulate and restrain their behavior. To train them irregularly for cultivating collective consciousness and hospital values, and so on. [8] To improve service quality, increase patient viscosity and increase numbers of outpatients. At the same time, team should create a positive and progressive corporate culture. The cohesion of all staff will promote the vigorous development of old Chinese medicine hospitals.

\section{B. To Build a Good Image with Integrated Marketing Strategy}

Today, marketing is not only the superposition of marketing personnel and market, but also the combination of strategy and marketing consciousness. If it only stays with the most superficial marketing means, the effect will be also limited. We should tend to diversify and establish hospital image through integrated marketing.[9]The reason why public hospitals are more favored and trusted by citizens lies not only in the policy support of the government, but also in their own public welfare activities and free consultation activities, such as post-disaster relief. Old TCM hospitals should take an active part in public welfare activities, so that people can change their stereotype towards private hospitals ; As well as to create a good image by word of mouth.

\section{To Improve Service Quality and Reduce Medical Disputes and Conflicts}

First, the old Chinese medicine hospital should establish the crisis management mechanism. So that when some medical disputes and other emergencies happened, problem can be solved timely. It also ensures that patients with minimal damage as well as protection of the interests of patients and a good old Chinese hospital image. In order to deal with these accidents properly, they can cooperate with the third party; It may amplify the good effect through the reports of the media 
and so on. It is also good for hospitals to find new development opportunities in the crisis. [10]

\section{Introducing Physician Resources to Infuse More New Blood}

Talent is another core problem in hospital competition. How can we establish an effective talent introduction and training system, strengthen talent training, and strengthen the core force without good medical resources and professional talents. In addition to the relevant policies issued by the state and the improvement of the hospital's own environment, the hospital should establish a corresponding system. It will attract talents and train and retain them.

It is impossible to win in competitions without a good physician resource and a professional talent pool. For establishing an effective system for the introduction and training of qualified personnel, we should strengthen personnel training, enhance the backbone, and improve the echelon of qualified personnel. Except for the relevant policies promulgated by the state and the improvement of the hospital's own environment, the hospital should also establish a corresponding system. Meanwhile, it can attract and cultivate talents. For the old traditional Chinese medicine hospital in Tianjin, firstly, it is necessary to establish a complete talent incentive system. It can ensure the stability of the talent echelon and improve the quality of the personnel; secondly, to establish the management idea, improve the reasonable treatment to the staff, and create a comfortable working atmosphere; thirdly, to strengthen the cohesion of the hospital, and to provide talent with a good platform through corporate culture.

\section{E. To Expand Business Channels, Add New Products, Guiding Diversification of the Hospital}

For example, self-made prescription medicine bag can increase the hospital business income. A clear classification of each department for the convenience to see doctors. To expand the scale of the old Chinese medicine hospital, increase inspection facilities, wards, and so on. Drawing on the experience from those excellent marketing modes of other private hospitals in Tianjin. Combination of medical and nursing should be carried out, the development should be diversified, the income should be increased, and the competitiveness of hospitals should be improved.

\section{F. Improving Cookers of Decoction}

In the investigation of patients' satisfaction, some patients think that the hospital takes medicine for so long. The taste of medicine is not good. So the decoction equipment needs to be improved. As the hospital is artificial decoction, the decoction equipment, a common stainless steel pot, is relatively old. The capacity of the pan is 2.7. Pan can fry 7 doses and 14 bags, and one bag is $198 \mathrm{ml}$. It takes a long time and wastes of manpower. Now it is mostly 4.5L electric casserole on the market. Medicine Decoction is convenient and fast, the medicine of one-off frying is better than that of stainless steel pan in old Chinese medicine hospital, and it is less trouble. It would be better to use electric casserole in the market for frying medicine.

\section{G. To Strengthen Publicity, Brand Building, and Brand Awareness of the Whole Staff}

We can make use of all kinds of media in the Wechat-era to propagate the TCM hospital and improve the popularity of the TCM hospital in Tianjin. In the promotion of WeChat, the original WeChat public platform should be revised and improved. First of all, to enrich background function introductory information, so that the attention is gained at a glance; from the aesthetic point of view, the original two functional keys should be changed into three by adding a functional choice in the public platform. It can help patients' registration. Furthermore, daily posts should be followed with the main line of soft text. For example, tweets arranged according to the International Day of the World and the 24 solar terms are arranged in an orderly way. If there is a change in the sitting time, it must be sent out in time for convenience. In the Weibo promotion, set up a special Weibo account, regularly propagate and guide on Weibo. Issue about the days, Medical information, and medical technology development and so on. For example, the advantages of acupuncture and massage can be reimbursed in hospitals, and people-benefit policies are published to the public for increasing the numbers of patients seeking medical treatment.

Making full use of propaganda tools such as search engines, radios, television, magazine, brochure etc. to advertise widely, frequently and appropriately; in some special periods, such as "AI Wei month", "Science Popularization week" and so on, free health education will increase the people's health consciousness. It does not blindly instill to the general audience. Instead, it should be guided to enhance people's attention to health and confidence in hospital medical technology.

\section{REFERENCES}

[1] Zhang Moning. On the New turn of people's livelihood Reform from the Perspective of the "two Conferences"[J]South Reviews ,2016,(4):37-43.

[2] Deng Li. Discussion on the Feasibility of entering the Medical and Health area of the Capital of the Society at present stage.

[3] Wang Fengnan. Research on the Development of Private Hospitals in China[D].Beijing: Beijing University of information science and technology.2014.46-47.

[4] Zhang Jianying, Wang Lu, He Qiang. SWOT Analysis on the Development of Private Hospitals in China[J].Chinese Hospital Management.2016,(10):37-39.

[5] Liu Yan, Zhu Qian. The present situation of the Development of Social Capital in running Medical treatment and its external factors[J].Chinese health resources.2014,(3):183-185.

[6] Wen Xueguo, Fang Zhiwu. Reform of Chinese Medical and Health System. 2014 2015[R].Beijing: social Sciences Literature Press.2014.19-27.

[7] Zhang Wenjing. The current situation of private hospitals[J].Technology and Life.2009,(1):130-131.

[8] Zhang Zhenzhong. Opinions of the State Council of the CPC Central Committee on Deepening the Reform of the Medical and Health System[J].China Dispensary. 2010, 29(4):243-248.

[9] Cao Chunming, Chen Biao. Adjustment of Private Hospital Management under the New Medical Reform Policy [J].Consume Guide., 2009,(13):58-59.

[10] Gu Xingong. Current situation and innovative Countermeasures of Drug Price Control system in China[M]Beijing. Social science literature press.,2014. 165-194. 